\title{
Preoperative Inflammatory Response to Esophageal Cancer Is More Reliable Prognostic Factor in Patients Received Esophagectomy
}

Yoshinori Fujiwara ( $\square$ yyfujiwara@nifty.cm )

Kawasaki Medical School

Shunji Endo

Kawasaki Medical School

Masaharu Higashida

Kawasaki Medical School

Hisako Kubota

Kawasaki Medical School

Seiya Kinoshita

Kawasaki Medical School

Kazuhiko Yoshimatsu

Kawasaki Medical School

Tomio Ueno

Kawasaki Medical School

\section{Research Article}

Keywords: mGPS, mCONUT, CRP, esophageal squamous cell carcinoma

Posted Date: December 29th, 2021

DOI: https://doi.org/10.21203/rs.3.rs-1184819/v1

License: (c) (1) This work is licensed under a Creative Commons Attribution 4.0 International License.

Read Full License 


\section{Abstract}

Background. Inflammation and nutrition are closely related to the progression of gastrointestinal malignancies. We aimed to explore the potential of preoperative inflammation-based or nutrition-based biomarkers as predictors of survival in patients with resectable esophageal squamous cell carcinoma (ESCC) using multivariate Cox analysis.

Methods. We included 122 patients with resectable ESCC (stages I-IV) in the study. We assessed the inflammation-based modified Glasgow prognostic score (mGPS), nutrition-based modified controlling nutritional status (mCONUT) score, CRP(C-reactive protein), serum albumin, lymphocyte counts, and total cholesterol. The relationships of these biomarkers with overall survival (OS) and recurrence-free survival (RFS) were evaluated. Three Cox model were performed for single parameters(CRP, albumin, lymphocyte, total cholesterol), for mCONUT and mGPS, and for clinicopathological factors.

Results. The cut-off values for CRP, albumin, and mCONUT measured using receiver operating characteristic (ROC) curves were $0.3,3.5$, and 4 , respectively. Patients with high mGPS and high mCONUT scores were significantly associated with shorter OS and RFS $(p<0.05)$.Multivariate Cox analysis showed that mGPS,pStage,tumor location were independent prognostic factors both FRS and OS. Also, Cox analysis for single parameters showed that preoperative CRP, lymphocyte counts were independent prognostic factors for RFS and albumin was prognostic factor for OS.

Conclusions. Preoperative inflammation-based mGPS is most reliable independent prognostic factor in patients with resectable ESCC. Suppression of preoperative inflammation can be improved nutritional status and may improve the prognosis in these patients.

\section{Introduction}

Esophageal cancer is the ninth most common cancer worldwide, with 604100 new cases the standard treatment for patients with locoregionally confined esophageal carcinoma. Despite curative surgery, a high recurrence rate is observed in patients with esophageal cancer (1-3). Perioperative chemotherapy and chemoradiotherapy have been developed to improve the survival of patients undergoing esophagectomy (4). Approximately $57 \%$ - $85 \%$ of patients with esophageal cancer have over $10 \%$ unintentional weight loss before treatment, which is associated with poor survival $(5,6)$.

An acute phase response (APR) leads to both inflammatory and neoplastic conditions (7), following the increased production of proinflammatory cytokines including IL-6 and TNF-a from tumor cells. These cytokines act on the liver to increase hepatic synthesis of the acute phase protein, C-reactive protein (CRP). Elevated CRP levels are associated with bodyweight loss and poor prognosis in gastrointestinal malignancies $(8,9)$. The modified Glasgow prognostic score (mGPS), an inflammation-based biomarker consisted of serum CRP and albumin levels, and it has been validated as an independent prognostic factor for various malignancies (10). On the other hand, the controlling nutritional status (CONUT) score is nutrition-based biomarker consisted of serum albumin, total cholesterol, and total lymphocyte counts, 
but not included inflammatory markers (11). It has been associated with postoperative complications and prognosis in several cancers (12-15). As prognostic markers associated with resectable esophageal cancer, the mGPS score was controversial for predicting survival, regardless of different cut-off values for each marker, while the CONUT score is a favorable predictor of survival, $(16,17)$. It is not clear whether preoperative these immunological, nutritional factors, inflammatory responses as a single marker, or their comprehensive markers determine the prognosis of patients with resectable esophageal cancer.

In the present study, we retrospectively reviewed patients who underwent curative surgery for esophageal squamous cell carcinoma (ESCC) in our department and attempted to identify the highest priority prognostic factors for ESCC patients after esophagectomy by evaluating these preoperative parameters using multivariate analysis.

\section{Patients And Methods}

We retrospectively reviewed 122 consecutive patients with biopsy-proven invasive ESCC who underwent esophagectomy with two or three-field lymphadenectomies at the Kawasaki Medical School Hospital between January 2011 and July 2020. Preoperative tumor stages were defined by upper GI ,endoscopy with biopsy, enhanced computed tomography, and positron emission tomography-computed tomography (PET-CT). Tumor stages were determined based on the eighth edition of the UICC classification of malignant tumors (18). Patients with R1/2 resections were excluded, and only those with R0 resections were evaluated. Neoadjuvant therapy consisting of 5-FU/cisplatin or 5FU/cisplatin/docetaxel was administered to seven cStagell/III patients after 2017. Seven patients of cStagell/III were received neoadjuvant therapy and esophagectomy was performed 3-7 weeks later after completion of chemotherapy. Three surgical procedures were performed. Ivor Lewis esophagectomy with two-or threefield lymph node dissections was performed before 2013. Esophageal reconstruction was performed using a gastric tube in the retrosternal or posterior mediastinal roots. However, cases after 2013 utilized thoracoscopic and laparoscopic-assisted approaches. Trans-hiatal esophagectomy with middle, lower mediastinal, and abdominal lymphadenectomy were also performed in the relatively early stages of lower esophageal cancer. Postoperative complications were evaluated according to the Clavien-Dindo classification grade 2 or higher (19). This study was approved by the Institutional Review Board of Kawasaki Medical School (Authorization No: 3382-2). Data were collected from the medical records at Kawasaki Medical School Hospital, and details of this retrospective study are published on the hospital home page. All patients were followed up regularly until July 2020 or until death. Informed consent was obtained from all the patients.

\section{Nutritional, immunological, and inflammatory response assessment}

Peripheral blood samples were collected for routine laboratory measurements of albumin and CRP within 1-4 weeks before surgery. While CRP was measured using anti-human CRP mouse monoclonal antibody sensitive latex (SEKISUI, Tokyo, Japan), serum albumin was measured using bromocresol purple 
(SEKISUI, Tokyo, Japan). Cholesterol was measured using the HMMPS method (FUJIFILM, Tokyo, Japan). The mGPS was determined as previously described based on the CRP and serum albumin levels (20). A time-dependent receiver operating characteristic (ROC) curve was calculated to evaluate the sensitivity and specificity of CRP and albumin in predicting 3-year recurrence-free survival (RFS). The Youden's index was estimated to determine the optimal cut-off for CRP and serum albumin (Fig. 1A and 1B). Based on these analyses, the optimal cut-off values for CRP and albumin were $0.3 \mathrm{mg} / \mathrm{dl}$ and 3.5 $\mathrm{g} / \mathrm{dl}$, respectively. The mGPS for esophageal cancer was calculated as follows: elevated $\operatorname{CRP}(>0.3$ $\mathrm{mg} / \mathrm{dl}$ ) with hypoalbuminemia $(<3.5 \mathrm{~g} / \mathrm{dl}$ ) was allocated a score of 2 (mGPS 2), while the presence of only one of these abnormalities was allocated a score of 1 (mGPS1). The absence of both these abnormalities was allocated a score of 0 (mGPSO). The mCONUT was calculated as per previously published criteria (11). As shown in Table 1, minimal changes were observed in serum albumin values. The mCONUT score, body mass index $\left(\mathrm{BMl}, \mathrm{kg} / \mathrm{m}^{2}\right)$ were evaluated. ROC curves estimated the cut-off values for these parameters for predicting 3-year RFS.

\section{Statistical analysis}

The chi-square or Fisher's exact t-test was used for categorical variables. The Mann-Whitney U test was used for continuous variables. When comparing three or more groups, the Holm method was used.

Overall survival (OS) was defined as the time between surgery and the patient's death or when the final information on vital status was available. RFS was defined as the time between surgery and cancer recurrence, death, or when the final information on vital status was available. The Kaplan-Meier method estimated survivals, and groups were compared using the two-sided log-rank test. Univariate and multivariate analyses for OS and RFS were performed using the Cox proportional hazard regression model, and survival with a 95\% confidence interval $(\mathrm{Cl})$ was determined. Results were considered significant at $p<0.05$. Three Cox models were used for nutritional, inflammatory and clinicopathological parameters, factors in the model were selected based on the following criteria: Age and gender was basically included, and (i) the number of explanatory variables were approximately $1 / 10$ the number of event occurrences, (ii) factors dependent on each other were not entered, (iii) useful independent prognostic factors were selected from previously published data.

Statistical analyses were performed using JMP (version 14; SAS, Tokyo, Japan) and R version 3.1.1 (R Project for Statistical Computing, Vienna, Austria).

\section{Results}

The mean $( \pm S D)$ follow-up time in this study was 38.02 $( \pm 29.91)$ months, and the median age of the patients was $66.6( \pm 7.59)$ years. The average BMI was $20.812( \pm 2.92) \mathrm{kg} / \mathrm{m}^{2}$. The cut-off values of BMI for RFS were 21.403 (sensitivity: 0.459 , specificity: 0.708 , and AUC: 0.522$) \mathrm{kg} / \mathrm{m} 2$. The preoperative CRP levels tended to be higher in the high BMI group than in the low BMI group ( $0.54 \mathrm{vs} .0 .32 \mathrm{mg} / \mathrm{dl}, \mathrm{p}=$ 0.073). The cut-off value of preoperative Cholesterol(/mg/dl) and Lymphocyte(/mm3) were 205 (sensitivity: 0.419, specificity: 0.75, and AUC: 0.576), 2270 (sensitivity: 0.257, specificity: 0.889, and AUC: 
0.545),respectively. Final pathological diagnosis of esophagectomy patients received neoadjuvant therapy were pStagell in 4 and pStagelll in 3 patients, respectively. Table 2 summarizes the relationship between the mGPS and clinicopathological features in patients with esophageal cancer. After excluding eight patients with no CRP data, 114 patients were evaluated. The proportion of patients younger than 70 was significantly higher in the mGPS 0 group $(p=0.035)$. Table 3 shows the relationship between the mCONUT scores and clinicopathological features in patients with esophageal cancer. After excluding seven patients with no lymphocyte data, 115 patients were evaluated. The number of patients with a moderate mCONUT score was significantly lower in the middle esophagus $(p=0.0023)$. None of the patients had severe mCONUT scores.

\section{Survival}

Fifty patients were cancer recurrence or death in observation period. The three-year OS and RFS for the entire population were $61.2 \%$ and $57.3 \%$, respectively, while the five-year OS and RFS were $58.4 \%$ and $57.1 \%$, respectively.

\section{Preoperative mGPS values for predicting OS and RFS after esophagectomy}

Kaplan Meier survival curves for DFS and OS in the different mGPS groups were analyzed. A high mGPS score was significantly associated with an unfavorable OS $(p=0.00357)$ and RFS $(p=0.00173)$. Compared to mGPS0, mGPS1 and mGPS2 were associated with significantly worse OS $(p=0.039$ and 0.004 , respectively; Fig. $2 A$ ) and RFS ( $p=0.0246$ and 0.0028 , respectively; Fig. 2B).

\section{Preoperative mCONUT values for predicting OS and RFS after esophagectomy}

There were no patients in the severe mCONUT group, and therefore, we compared the three groups, (Normal,Mild,Severe). Based on the ROC curve, the cut-off mCONUT value was 4 (sensitivity: 0.289, specificity: 0.857, AUC: 0.542). and patients were divided into low and high mCONUT groups. Figures $3 \mathrm{~A}$ and $3 \mathrm{~B}$ show the Kaplan-Meier OS and RFS curves for the different mCONUT groups. The low mCONUT group had significantly better OS and RFS than the high mCONUT group ( $p=0.0349,0.0351$, respectively ) .

\section{Relationship between serum Albumin and CRP}

Relationship between preoperative serum Albumin and CRP level was shown in Figure4. Serum Albumin level was tendency to be higher in low $\mathrm{CRP}(<0.3 \mathrm{mg} / \mathrm{dl}$ ) group(Medan value, $4.05 \mathrm{vs} 3.9 \mathrm{mg} / \mathrm{dl}, \mathrm{p}=0.079$ ).

\section{Univariate and multivariate Cox analysis for OS and RFS}

Univariate analysis revealed that TNM stage, depth of tumor invasion, tumor location, Albumin,CRP, mGPS, and mCONUT were significant prognostic factors for both OS and RFS in patients with resectable ESCC. (Table 4). Cox proportional hazard model for single nutritional and inflammatory covariates 
showed preoperative CRP and Lymphocyte counts were independent prognostic factors for DFS, and preoperative albumin level was independent prognostic factor for OS(Table5). As the comprehensive biomarkers,Cox analysis showed that only mGPS emerged as independent prognostic factors for OS and RFS(Table6). Cox analysis for clinicopathological covariates and mGPS showed that pStages, tumor locations, and mGPS were independent prognostic factors for both RFS and OS(Table7).

\section{Discussion}

This is the first multivariate analysis of whether the preoperative inflammatory-based biomarker (mGPS) or the nutritional-based biomarker( $\mathrm{mCONUT}$ ) can be most reliable prognostic factors in patients with resectable ESCC. We have demonstrated that the mGPS score was an independent prognostic factor for both OS and RFS in Cox analysis. Namely, inflammatory-based comprehensive biomarker represented by CRP and albumin predict postoperative cancer recurrence and survival. Another Cox analysis showed that preoperative CRP predicted the cancer recurrence. As the cut off value of CRP level in GPS, O'Gorman et al. set it at $1 \mathrm{mg} / \mathrm{dL}(21)$. In the present study, using the ROC curve to estimate RFS, we set the cut-off value at $0.3 \mathrm{mg} / \mathrm{dL}$, which was consistent with the Japanese standard CRP value before 2019 (22). As per the patient care and health information from Mayo Clinic, the normal CRP level is $<10 \mathrm{mg} / \mathrm{L}$, and $>10$ $\mathrm{mg} / \mathrm{L}$ is a sign of serious infection, trauma, or chronic disease (23). Obesity is also associated with elevated CRP levels, probably due to the abundance of proinflammatory mediators such as TNF- $\alpha$ and IL6 in adipose tissues (24).The standard CRP values in Japan were inconsistent with those in other countries. Therefore, the standard CRP value of $\geq 1 \mathrm{mg} / \mathrm{dl}$ is not suitable for Japanese patients with a mean BMI of $<25 \mathrm{~kg} / \mathrm{m}^{2}(25)$. A meta-analysis showed that preoperative high CRP levels were associated with poor OS in esophageal SCC patients received surgery(26), but there was little reports association between preoperative CRP and RFS regarding esophagectomy. In the present study, preoperative high CRP level can predict recurrence of esophageal cancer after esophagectomy, this might be the result of the preoperative high CRP status persisting during the perioperative period and continued to activation of cancer cells(27). Preoperative low serum albumin level as an indicator of nutritional status was associated with poor OS in Cox analysis. It was possibly that preoperative albumin level also affected the postoperative nutritional status, and it affected the response to treatment and increased the risk of chemotherapy induced toxicity at the time of recurrences(28).

The GPS score was first introduced by Forrest et al. in 2003 as a prognostic marker for inoperable nonsmall cell lung cancer [20]. There are seven retrospective reports before 2020 on the association between mGPS scores and esophageal surgery, all from Asian countries, including Japan and China (29-35). The mGPS scores for prognostic factors in esophageal cancer patients after esophagectomy are controversial, might be the different cut-off value of CRP. In the present study, we found that the mCONUT score could not be a prognostic factor for OS and RFS in Cox analysis. The parameter common to both the mCONUT score and mGPS is serum albumin. The major difference between mGPS and mCOUNT is whether the CRP value is included. As mentioned above, we showed that preoperative CRP level was important biomarker for predicting cancer recurrence after esophagectomy, and albumin level was 
independent prognostic factor for OS in Cox analysis. In general, CRP and albumin might be linked together, and hypoalbuminemia reflects the presence of systemic inflammation $(36,37)$. This phenomenon was also recognized in this study (Figure4). Scot et al. reported an increase in the systemic inflammatory response associated with weight loss, reduced albumin concentration, reduced performance status, and reduced survival in patients with inoperable non-small-cell lung cancer (37). Cancer-induced cachexia, a guideline for the oncologist, states that proinflammatory cytokines such as IL-1, 6 and TNFa are released as a local response to the tumor cells. These cytokines act on the liver and increase hepatic synthesis of acute-phase proteins such as CRP and fibrinogen, inducing muscle proteolysis, fat lipolysis, and cancer-related cachexia(38). Thus, pretreatment positive CRP levels lead to worse outcomes in cancer patients. As a nutritional intervention for cancer-induced cachexia, positive CRP levels may prevent the improvement of nutritional status. Nutritional interventions for patients with cancer are controversial. Recent ESPEN(European Society for Clinical Nutrition and Metabolism) guidelines showed that preoperative nutritional intervention for patients of severe malnutrition received major surgery was effective to reduced postoperative surgical site infection. But there was no description of the relationship between nutritional intervention and prognosis(39). On the other hand, Gullett et al. showed that nutritional intervention does not recommend in patients undergoing major cancer surgery for routine use (40). They also classified the wasting syndromes into three categories(starvation, cachexia and sarcopenia) in cancer patients and reported that nutritional intervention might be effect in these patients with starvation (38). Based on these findings, it seems reasonable that patients with improved mGPS score during NAC(Neoadjuvant chemotherapy) had a better prognosis, although the number of cases was small, as reported by Otowa et al. (31). To improve survival in resectable esophageal cancer patients, preoperative chemotherapy or chemoradiotherapy that results in reduced CRP levels may be effective and improve the patient's nutritional status. Unfortunately, we could not evaluated the NAC patients because of small number of patients in this study.

This study has some limitations. First, it was a retrospective study and included data from a single institution. Large retrospective cohort studies might be necessary for the future to accurately evaluate the role of preoperative inflammatory, nutritional, and immunological factors as predictors of survival in patients with resectable esophageal cancer.

In conclusion, this study suggests that preoperative mGPS score is an independent prognostic factor in patients with resectable esophageal cancer. CRP and albumin levels are simple and easy to measure in clinical practice. More effective preoperative therapies and nutritional interventions which lead to negative CRP levels might be necessary to improve survival in these patients.

\section{Abbreviations}

C-reactive protein (CRP), modified Glasgow prognostic score (mGPS), the controlling nutritional status (CONUT), overall survival (OS), recurrence-free survival (RFS), esophageal squamous cell carcinoma (ESCC), receiver operating characteristic (ROC), body mass index (BMI) 


\section{Disclosures And Declarations}

1, Ethical approval: All procedures performed in studies involving human participants were in accordance with the ethical standards of the institutional and/or national research committee and with the 1964 Helsinki declaration and its later amendments or comparable ethical standards.

ETHICAL APPROVAL

The Research Ethics Committee(REC) of Kawasaki Medical School

Protocol number: Authorization No: 3382-2

Dean of Kawasaki Medical School

Masao Fukunaga, M.D., Ph.D.

2, Informed consent: Informed consent was obtained from all individual participants included in the study, and it was obtained in writing prior to surgery.

3, Consent for publication: Not applicable.

4, Disclosure of potential conflicts of interest: No COI (conflicts of interest) disclosure of all authors in this study.

5 , No funding of this study in all authors.

6, Availability of data and materials

The datasets generated and/or analysed during the current study are not publicly available because permission of our hospital and university were not given, but are available from the corresponding author on reasonable request.

7, Acknowledgements

We would like to thank Miss Saki and Mrs. Kimura for their assistance in collecting data.

Authors contributions

'YF' and 'SE' :Statistical analysis and wrote Introduction, Methods and Discussion.

'MH' 'SK'and 'HK': Assisted data collections and clinical practice. Figure and Table formation.

'KY': assisted statistical analysis and comprehensive check.

'TU': Final check of manuscript and suggested appropriate corrections.

All authors have read and approved the manuscript for submission. 


\section{References}

1. Mariette C, Balon JM, Piessen G, Fabre S, Van Seuningen I, Triboulet JP. Pattern of recurrence following complete resection of esophageal carcinoma and factors predictive of recurrent disease. Cancer. 2003;97(7):1616-23.

2. Hulscher JB, van Sandick JW, Tijssen JG, Obertop H, van Lanschot JJ. The recurrence pattern of esophageal carcinoma after transhiatal resection. J Am Coll Surg. 2000;191(2):143-8.

3. Nakagawa S, Kanda T, Kosugi S, Ohashi M, Suzuki T, Hatakeyama K. Recurrence pattern of squamous cell carcinoma of the thoracic esophagus after extended radical esophagectomy with three-field lymphadenectomy. J Am Coll Surg. 2004;198(2):205-11.

4. Xiao X, Hong HG, Zeng X, Yang YS, Luan SY, Li Y, et al. The Efficacy of Neoadjuvant Versus Adjuvant Therapy for Resectable Esophageal Cancer Patients: A Systematic Review and Meta-Analysis. World journal of surgery. 2020;44(12):4161-74.

5. Book chapter;Barrett T,Nutritional Support in Esophageal Cancer In:Nabil F.S,Bassel F.EL-R,editors. Esophageaal Cancer.Springer Nature Switzerland AG;2020.pp323-336.

6. Zhang S, Tan Y, Cai X, Luo K, Wu Z, Lu J. Preoperative weight loss is associated with poorer prognosis in operable esophageal cancer patients: A single-center retrospective analysis of a large cohort of Chinese patients. Journal of Cancer. 2020;11(7):1994-9.

7. Deans C, Wigmore SJ. Systemic inflammation, cachexia and prognosis in patients with cancer. Current opinion in clinical nutrition and metabolic care. 2005;8(3):265-9.

8. McMillan DC, Elahi MM, Sattar N, Angerson WJ, Johnstone J, McArdle CS. Measurement of the systemic inflammatory response predicts cancer-specific and non-cancer survival in patients with cancer. Nutrition and cancer. 2001;41(1-2):64-9.

9. Barber MD, Ross JA, Fearon KC. Changes in nutritional, functional, and inflammatory markers in advanced pancreatic cancer. Nutrition and cancer. 1999;35(2):106-10.

10. McMillan DC. The systemic inflammation-based Glasgow Prognostic Score: a decade of experience in patients with cancer. Cancer treatment reviews. 2013;39(5):534-40.

11. Ignacio de Ulíbarri J, González-Madroño A, de Villar NG, González P, González B, Mancha A, et al. CONUT: a tool for controlling nutritional status. First validation in a hospital population. Nutricion hospitalaria. 2005;20(1):38-45.

12. Yoshida N, Baba Y, Shigaki H, Harada K, Iwatsuki M, Kurashige J, et al. Preoperative Nutritional Assessment by Controlling Nutritional Status (CONUT) is Useful to estimate Postoperative Morbidity After Esophagectomy for Esophageal Cancer. World journal of surgery. 2016;40(8):1910-7.

13. Tokunaga R, Sakamoto Y, Nakagawa S, Ohuchi M, Izumi D, Kosumi K, et al. CONUT: a novel independent predictive score for colorectal cancer patients undergoing potentially curative resection. International journal of colorectal disease. 2017;32(1):99-106.

14. Toyokawa T, Kubo N, Tamura T, Sakurai K, Amano R, Tanaka H, et al. The pretreatment Controlling Nutritional Status (CONUT) score is an independent prognostic factor in patients with resectable 
thoracic esophageal squamous cell carcinoma: results from a retrospective study. BMC cancer. 2016;16(1):722.

15. Iseki Y, Shibutani M, Maeda K, Nagahara H, Ohtani H, Sugano K, et al. Impact of the Preoperative Controlling Nutritional Status (CONUT) Score on the Survival after Curative Surgery for Colorectal Cancer. PloS one. 2015;10(7):e0132488.

16. Takagi K, Buettner S, ljzermans JNM, Wijnhoven BPL. Systematic Review on the Controlling Nutritional Status (CONUT) Score in Patients Undergoing Esophagectomy for Esophageal Cancer. Anticancer research. 2020;40(10):5343-9.

17. Wang Y, Chen L, Wu Y, Li P, Che G. The prognostic value of modified Glasgow prognostic score in patients with esophageal squamous cell cancer: a Meta-analysis. Nutrition and cancer. 2020;72(7):1146-54.

18. James D.Brierley MKGCW. UICC :TNM Classification of MALIGNAT TUMORS Eighth Edition: WILEY Blackwell ,Kanehara,Tokyo,Japan; 2017.

19. Katayama H, Kurokawa Y, Nakamura K, Ito H, Kanemitsu Y, Masuda N, et al. Extended Clavien-Dindo classification of surgical complications: Japan Clinical Oncology Group postoperative complications criteria. Surgery today. 2016;46(6):668-85.

20. Forrest LM, McMillan DC, McArdle CS, Angerson WJ, Dunlop DJ. Evaluation of cumulative prognostic scores based on the systemic inflammatory response in patients with inoperable non-small-cell lung cancer. British journal of cancer. 2003;89(6):1028-30.

21. O'Gorman P, McMillan DC, McArdle CS. Prognostic factors in advanced gastrointestinal cancer patients with weight loss. Nutrition and cancer. 2000;37(1):36-40.

22. Tani N. C-reactive protein(CRP). Nippon Rinsho. 2004;62(Suppl11): 212-216 in Japanese.

23. Online document; MAYO CLINIC.Patient Care \& Health Information Tests \& Procedures. In:C-reactive protein test. https://www.mayoclinic.org/tests-procedures/c-reactive-protein-test/about/pac20385228 Accessed 24 May 2021.

24. Lau DCW, Dhillon B, Yan H, Szmitko PE, Verma S. Adipokines: molecular links between obesity and atherosicerosis. American Journal of Physiology-Heart and Circulatory Physiology. 2005;288(5):H2031-H41.

25. Afshin A, Forouzanfar MH, Reitsma MB, Sur P, Estep K, Lee A, et al. Health Effects of Overweight and Obesity in 195 Countries over 25 Years. The New England journal of medicine. 2017;377(1):13-27.

26. Huang Y, Feng JF, Liu JS, Chen QX. Prognostic role of serum C-reactive protein in esophageal cancer: a systematic review and meta-analysis. Therapeutics and clinical risk management. 2015;11:89-94.

27. Guillem P, Triboulet JP. Elevated serum levels of C-reactive protein are indicative of a poor prognosis in patients with esophageal cancer. Diseases of the esophagus : official journal of the International Society for Diseases of the Esophagus. 2005;18(3):146-50.

28. Gupta D, Lis CG. Pretreatment serum albumin as a predictor of cancer survival: a systematic review of the epidemiological literature. Nutrition journal. 2010;9:69. 
29. Tian R, Zhang F, Sun P, Wu J, Yan H, Wu AR, et al. The preoperative sensitive-modified Glasgow prognostic score is superior to the modified Glasgow prognostic score in predicting long-term survival for esophageal squamous cell carcinoma. Oncotarget. 2016;7(41):67485-94.

30. Tan Z, Zhang M, Han Q, Wen J, Luo K, Lin P, et al. A novel blood tool of cancer prognosis in esophageal squamous cell carcinoma: the Fibrinogen/Albumin Ratio. Journal of Cancer. 2017;8(6):1025-9.

31. Otowa Y, Nakamura T, Takiguchi G, Tomono A, Yamamoto M, Kanaji S, et al. Changes in modified Glasgow prognostic score after neoadjuvant chemotherapy is a prognostic factor in clinical stage II/III esophageal cancer. Diseases of the esophagus : official journal of the International Society for Diseases of the Esophagus. 2016;29(2):146-51.

32. Arigami T, Okumura $H$, Matsumoto $M$, Uchikado $Y$, Uenosono $Y$, Kita $Y$, et al. Analysis of the Fibrinogen and Neutrophil-Lymphocyte Ratio in Esophageal Squamous Cell Carcinoma: A Promising Blood Marker of Tumor Progression and Prognosis. Medicine. 2015;94(42):e1702.

33. Matsuda S, Takeuchi H, Kawakubo H, Fukuda K, Nakamura R, Takahashi T, et al. Correlation Between Intense Postoperative Inflammatory Response and Survival of Esophageal Cancer Patients Who Underwent Transthoracic Esophagectomy. Annals of surgical oncology. 2015;22(13):4453-60.

34. Hirahara N, Matsubara T, Hayashi H, Takai K, Fujii Y, Tajima Y. Impact of inflammation-based prognostic score on survival after curative thoracoscopic esophagectomy for esophageal cancer. European journal of surgical oncology : the journal of the European Society of Surgical Oncology and the British Association of Surgical Oncology. 2015;41(10):1308-15.

35. Yu X, Wen Y, Lin Y, Zhang X, Chen Y, Wang W, et al. The value of preoperative Glasgow Prognostic Score and the C-Reactive Protein to Albumin Ratio as prognostic factors for long-term survival in pathological T1N0 esophageal squamous cell carcinoma. Journal of Cancer. 2018;9(5):807-15.

36. Deans DA, Tan BH, Wigmore SJ, Ross JA, de Beaux AC, Paterson-Brown S, et al. The influence of systemic inflammation, dietary intake and stage of disease on rate of weight loss in patients with gastro-oesophageal cancer. British journal of cancer. 2009;100(1):63-9.

37. Scott HR, McMillan DC, Forrest LM, Brown DJ, McArdle CS, Milroy R. The systemic inflammatory response, weight loss, performance status and survival in patients with inoperable non-small cell lung cancer. British journal of cancer. 2002;87(3):264-7.

38. Gullett N, Rossi P, Kucuk O, Johnstone PA. Cancer-induced cachexia: a guide for the oncologist. Journal of the Society for Integrative Oncology. 2009;7(4):155-69.

39. Weimann A, Braga M, Carli F, Higashiguchi T, Hübner M, Klek S, et al. ESPEN practical guideline: Clinical nutrition in surgery. Clinical nutrition (Edinburgh, Scotland). 2021;40(7):4745-61.

40. Gullett NP, Mazurak VC, Hebbar G, Ziegler TR. Nutritional interventions for cancer-induced cachexia. Current problems in cancer. 2011;35(2):58-90.

\section{Tables}


Due to technical limitations, tables 1 to 7 xlsx are only available as a download in the Supplemental Files section.

\section{Figures}

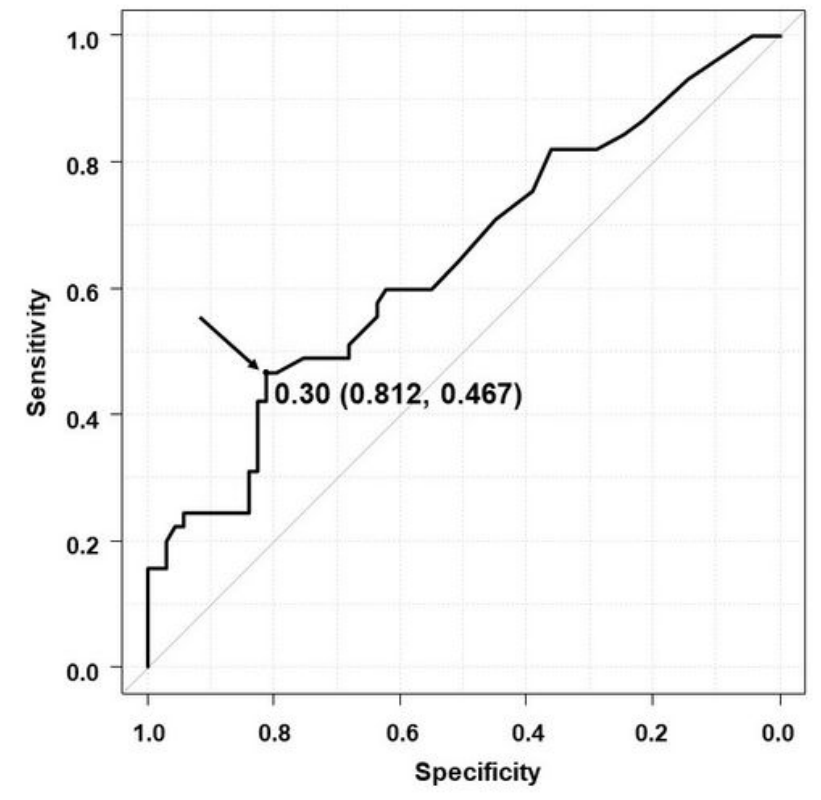

$1 \mathrm{~A}$

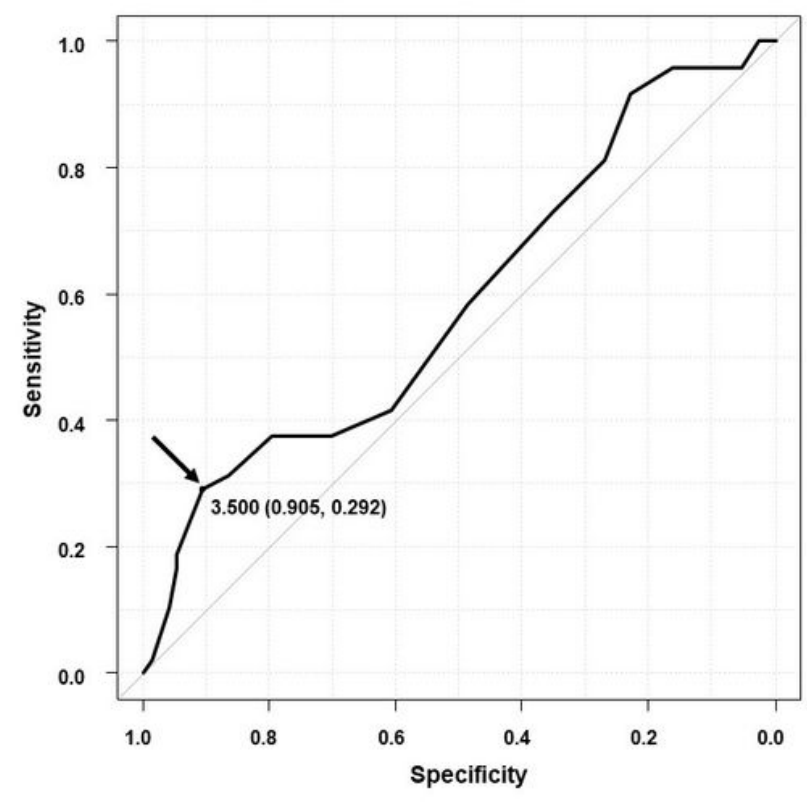

$1 \mathrm{~B}$

\section{Figure 1}

Receiver operating characteristics (ROC) curves

Shown are the ROC curves for (A) CRP (mg/dl) with a cut-off value of $0.3 \mathrm{mg} / \mathrm{dl}$; sensitivity, 0.467 ;

specificity, 0.812 , and AUC, 0.643 , and (B) albumin ( $\mathrm{g} / \mathrm{dl}$ ) with a cut off value of $3.5 \mathrm{~g} / \mathrm{dl}$; sensitivity, 0.292; specificity, 0.905; and AUC, 0.589 . 


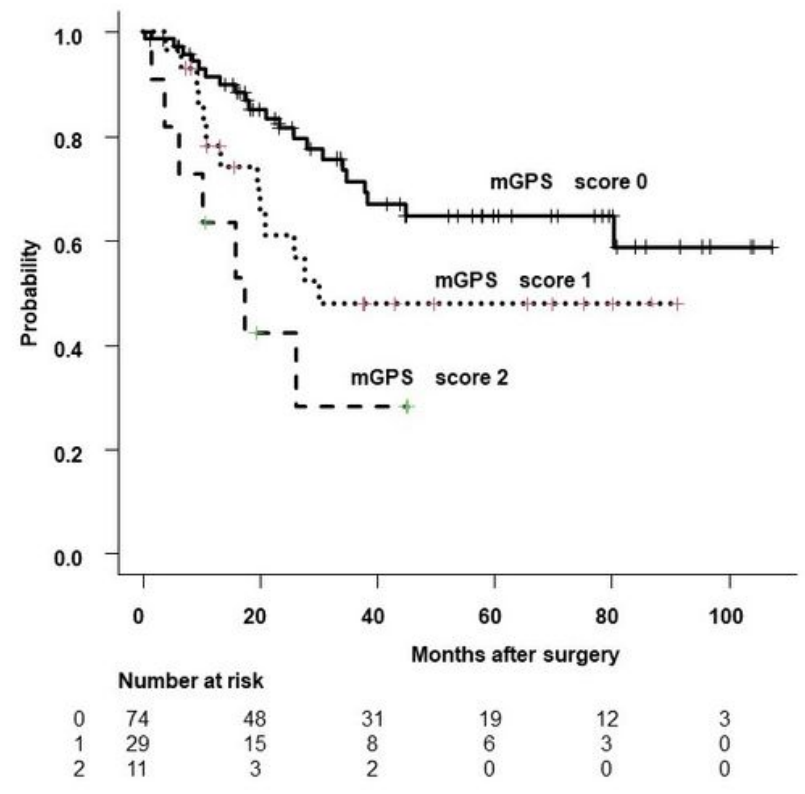

$2 \mathrm{~A}$

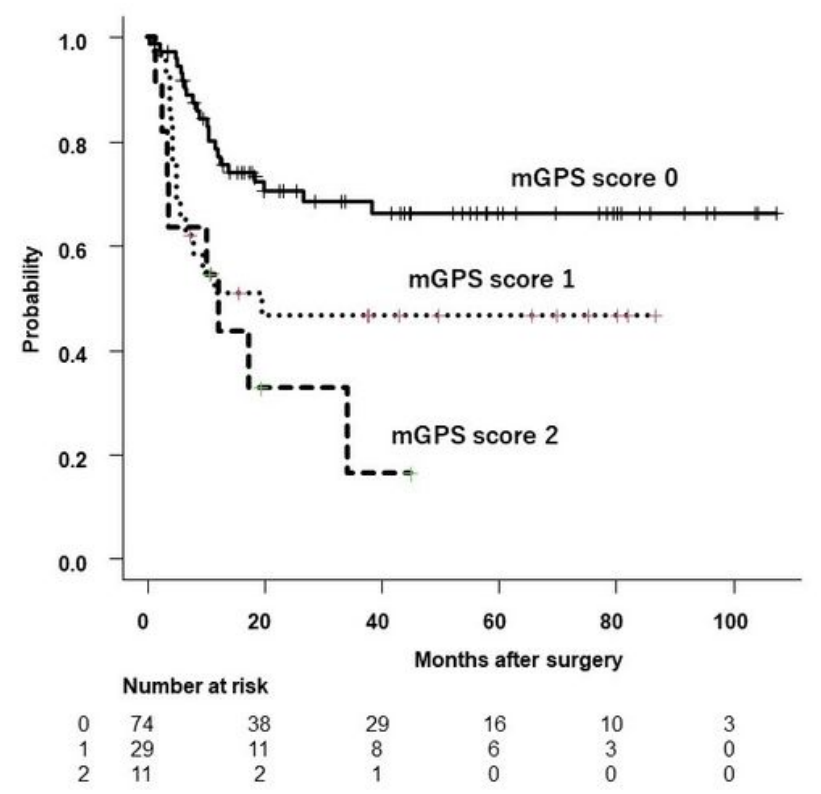

2B

\section{Figure 2}

2A Overall survival between different mGPS score in ESCC patients after esophagectomy. A high mGPS score was significantly associated with an unfavorable OS $(p=0.00357)$. ESCC: esophageal squamous cell carcinoma

2B Recurrence free survival between different mGPS score in ESCC patients after esophagectomy. A high mGPS score was significantly associated with an unfavorable RFS $(p=0.00173)$. ESCC: esophageal squamous cell carcinoma 


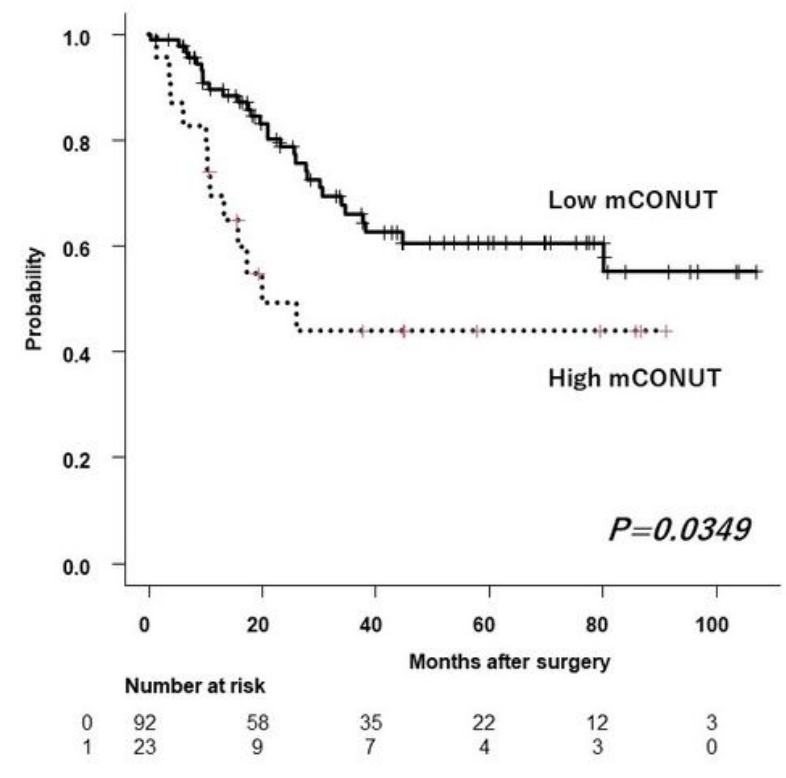

$3 A$

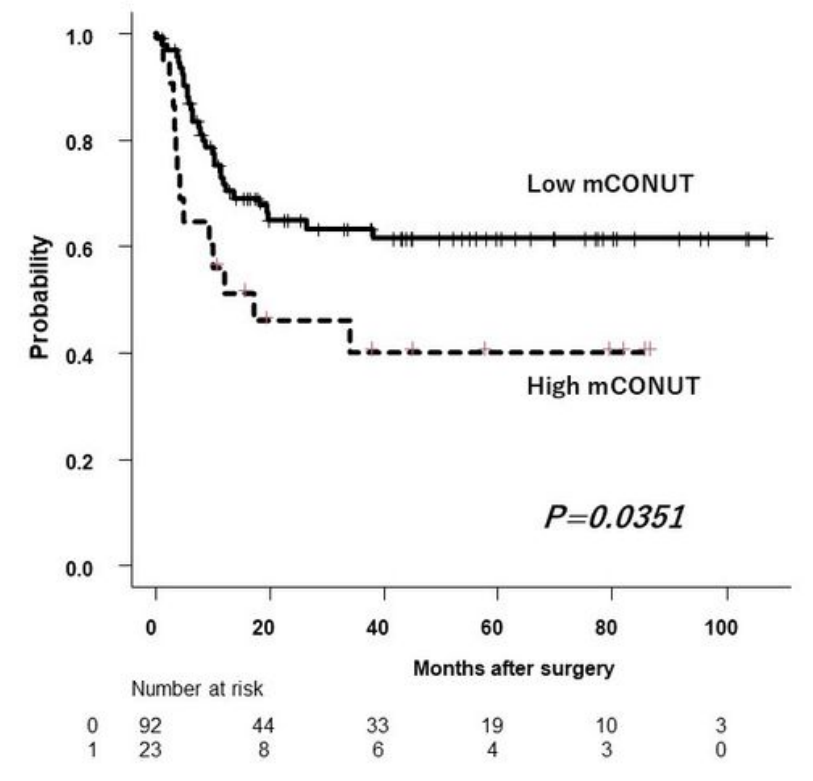

3B

\section{Figure 3}

Kaplan Meier survival curves in ESCC patients after esophagectomy based on mCONUT scores.

(A) The five- and three-year OS were $66 \%$ and $61.3 \%$, respectively, in the low mCONUT group and $43.8 \%, 43.8 \%$ in the high mCONUT group. The low mCONUT group showed significantly better OS than the high mCONUT group $(p<0.0349)$.

(B) The low mCONUT group showed significantly better RFS than the high mCONUT group $(p<0.0351)$. The five- and three-year RFS were $63.2 \%$ and $60.6 \%$, respectively, for the low mCONUT group and $40.8 \%$ , $40.8 \%$ for the high mCONUT group.

ESCC: esophageal squamous cell carcinoma; OS: Overall survival; RFS: Recurrence-free survival 


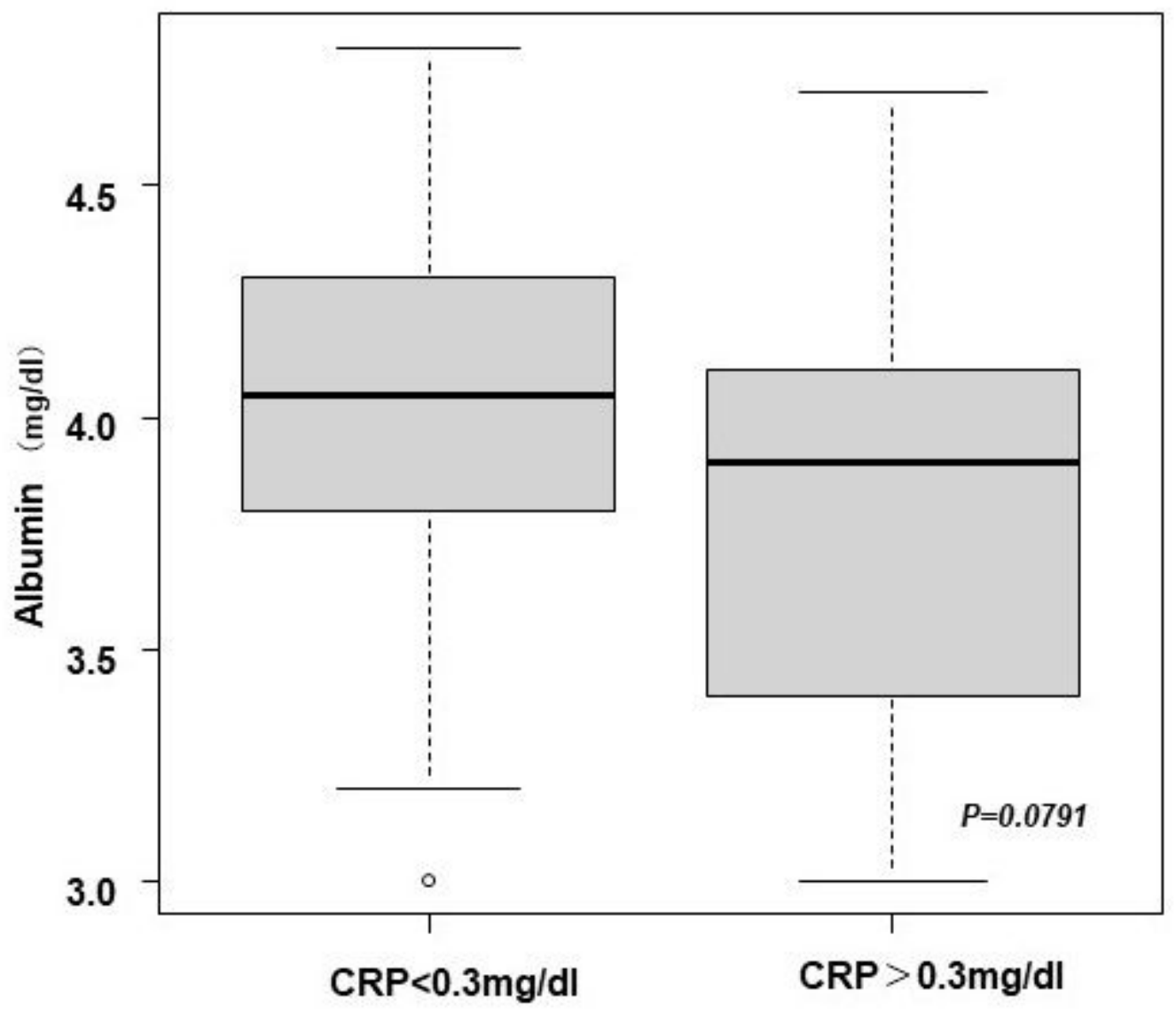

Figure 4

Preoperative serum albumin level and inflammatory response.

Serum Albumin level was tendency to be higher in low CRP $(<0.3 \mathrm{mg} / \mathrm{dl})$ group(Medan value,4.05 vs $3.9 \mathrm{mg} / \mathrm{dl}, \mathrm{p}=0.079)$.

\section{Supplementary Files}

This is a list of supplementary files associated with this preprint. Click to download.

- Table1.xlsx

- Table.xIsx

- Table3.xlsx

- Table4.xlsx 
- Table5.xIsx

- Table6.xlsx

- Table7.xlsx 